\title{
Generator-level studies for photon-tagged jet measurements and effects of angular resolution driven by heavy-ion background
}

\author{
Kaya Tatar* \\ Massachusetts Institute of Technology, Department of Physics \\ E-mail: tatarkemit.edu
}

\begin{abstract}
Photon+jet events are generated using PYTHIA 8 and their fraction of quark-jet and gluon-jet events are studied by mapping the photon+jet production to parton densities. Measurements had shown that photon+jet momentum imbalance and azimuthal angle correlations have wide distributions even in the absence of a QCD medium. Sources of widening are investigated via parton level processes in PYTHIA 8, finding that initial-state radiation is the dominant source. Correlations at RHIC energies are shown to have sharper distributions than those at LHC energies, as the effects of vacuum processes become smaller with lower collision energy. Sensitivity of jet observables to the heavy-ion underlying event (UE) are studied using toy events. Jet observables are compared between jets recombined with $E$-scheme and winner-take-all (WTA) schemes. The jet pointing angle in WTA scheme is found to be much less sensitive to the heavy-ion UE.
\end{abstract}

13th International Workshop in High pT Physics in the RHIC and LHC Era (High-pT2019)

19-22 March 2019

Knoxville, Tennessee, USA

\footnotetext{
* Speaker.
} 


\section{1. $\gamma$-tagged jet measurements at the LHC}

Hard scatterings that produce a parton recoiling from an electroweak (EW) boson are precise probes for studying the modifications of partons in a hot QCD medium. As EW bosons do not undergo strong interaction with the QCD matter, the kinematics of a parton before interacting with the medium can be controlled by tagging jets with photons or $\mathrm{Z}$ bosons. The CMS and ATLAS experiments at the LHC have performed several $\gamma$-tagged and Z-tagged jet measurements to quantify the modification of hard partons inside the hot QCD medium by comparing the results from $\mathrm{PbPb}$ collisions to the reference from pp collisions. Comparisons of the $\gamma+$ jet transverse momentum $\left(p_{\mathrm{T}}\right)$ imbalance, $x_{\mathrm{j} \gamma}=p_{\mathrm{T}}^{\text {jet }} / p_{\mathrm{T}}^{\gamma}$, suggest that jets with $p_{\mathrm{T}}^{\text {jet }} \sim 60 \mathrm{GeV} / \mathrm{c}$ lose $\sim 10 \%$ of their energy in $\mathrm{PbPb}$ collisions, as reported in Refs. [1, 2,3]. On the other hand, no significant modification has been observed in distributions of azimuthal angle difference, $\Delta \phi_{\mathrm{j} \gamma}=\left|\phi^{\text {jet }}-\phi^{\gamma}\right|$, between the photon and jets. Studies of Z-tagged jets yielded the same conclusions [4]. These measurements correlate the photon and jet kinematics using "bulk" observables which do not consider the jet internal structure. Later measurements analyzed the $\gamma$-tagged jet substructure. Fragmentation functions results $[5,6]$ unambiguously showed that parton showers are modified such that there are more low- $p_{\text {T }}$ particles and less high- $p_{\mathrm{T}}$ particles inside the jet cone. The jet radial momentum density profile (the jet shape) is distorted such that a larger fraction of jet energy is carried at large distance from the jet axis [7], consistent with the dijet and inclusive jet results [8, 9, 10, 11].

One feature of bulk observables is that the distributions of momentum $\left(x_{\mathrm{j} \gamma}\right)$ and azimuthal angle $\left(\Delta \phi_{\mathrm{j} \gamma}\right)$ correlations are fairly wide already in pp collisions where the medium effects are negligible. Two hard scatterers recoiling off of each other are tightly correlated in momentum and azimuthal angle because of momentum conservation. However, correlations of photons and jets are smeared into wide distributions and this widening is so large that it cannot be explained solely by the resolution of the experimental apparatus.

Jet measurements in heavy-ion collisions can be significantly distorted by uncorrelated particles from the underlying event (UE). Effects of the UE can be removed if the magnitude and direction of the effects on the measured quantity can be estimated. Techniques are established to remove the UE for scalar quantities for which the direction of effects are known a priori. For example, the UE increases the energy density, therefore its effects can be removed by subtracting the estimated energy density originating only from the UE. Another example is particle yield where the UE increases the number of particles; hence, the direction of the effects is known and the magnitude of the increase can be estimated using event-mixing techniques [5]. However, removing the effects of UE on vector quantities, such as position in $\eta-\phi$ plane, is more challenging, as the direction of the effect is not known a priori. For example, UE particles can distort the pointing angle of a reconstructed jet, but the direction of this distortion cannot be inferred without identifying the particles from the UE. This effect is crucial for observables that are sensitive to the jet $(\eta, \phi)$ coordinate, e.g. jet shapes.

This document presents results from event generator studies that address the items raised in the above two paragraphs. First, the physics processes that cause the widening of $\gamma+$ jet correlations are investigated using PYTHIA 8 [12] events. Second, the UE sensitivity of jet fragmentation functions and jet shapes are studied by embedding the PYTHIA 8 events into toy heavy-ion events. 


\section{2. $\gamma+$ jet events in PYTHIA 8}

PYTHIA 8 is chosen to generate the $\gamma+$ jet events, since it implements full event evolution from hard scattering to hadronization and provides a reasonably well description of the $\gamma$-tagged jet results in pp collisions $[2,3,5,7]$. Events are generated by activating the PromptPhoton: al 1 process (all prompt photon processes). The $q(\bar{q})+g \rightarrow \gamma+q(\bar{q})$ subprocess, known as the QCD Compton scattering, gives the dominant contribution to quark-jet events (events where the parton recoiling from the photon is a quark), and the $q+\bar{q} \rightarrow \gamma+g$ subprocess, namely the $q \bar{q}$ annihilation, is the dominant process for gluon-jet production. Fig. 1 shows the fraction of quark and gluon-jet events as a function of $p_{\mathrm{T}}^{\gamma}$ for a center-of-mass energy at the LHC (pp, $\left.\sqrt{s}=5.02 \mathrm{TeV}\right)$. The events are mostly quark-jet events with a fraction of about $90 \%$ for $p_{\mathrm{T}}^{\gamma} \sim 50 \mathrm{GeV} / \mathrm{c}$ which decreases to about $80 \%$ for $p_{\mathrm{T}}^{\gamma} \sim 1 \mathrm{TeV} / \mathrm{c}$. The evolution of the event fractions can be understood by examining the densities probed for the partons that initiate the scattering (incoming partons).

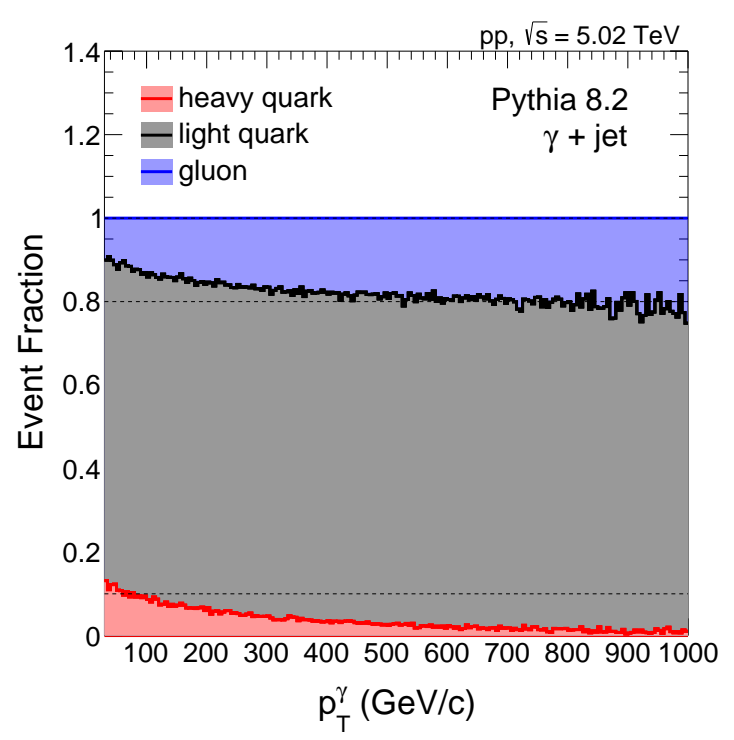

Figure 1: Prompt $\gamma+$ jet events are generated using PYTHIA 8 for pp collisions at $\sqrt{s}=5.02 \mathrm{TeV}$. The fraction of events for which the recoiling parton is a gluon, a light quark $(u, d, s)$, or a heavy quark $(c, b, t)$ is indicated with blue, gray, and red areas, respectively.

Fig. 2 shows the parton density $x \cdot f\left(x, Q^{2}\right)$ versus momentum fraction $x$ of incoming partons contributing to prompt photon processes in PYTHIA 8. Contributions to processes with large momentum transfer $Q$ are from partons with large $x$. In addition, gluon densities decrease faster with $x$ than quark densities. Therefore, relative contribution of gluon initiated processes decrease with increasing $Q$. Since quark-jets are initiated dominantly by gluons, their event fraction decreases with increasing $Q$ or equivalently with increasing $p_{\mathrm{T}}$.

Parton densities can also help to understand how the $\gamma+$ jet events differ for different collision energies. The left panel of Fig. 3 shows the $\gamma+$ jet event fractions for a center-of-mass energy at the RHIC (pp, $\sqrt{s}=200 \mathrm{GeV}$ ). The quark-jet fraction decreases dramatically after $p_{\mathrm{T}}^{\gamma} \sim 50 \mathrm{GeV} / \mathrm{c}$. Almost all $\gamma+$ jet production at RHIC energies is originating from the region with high $x$, in par- 

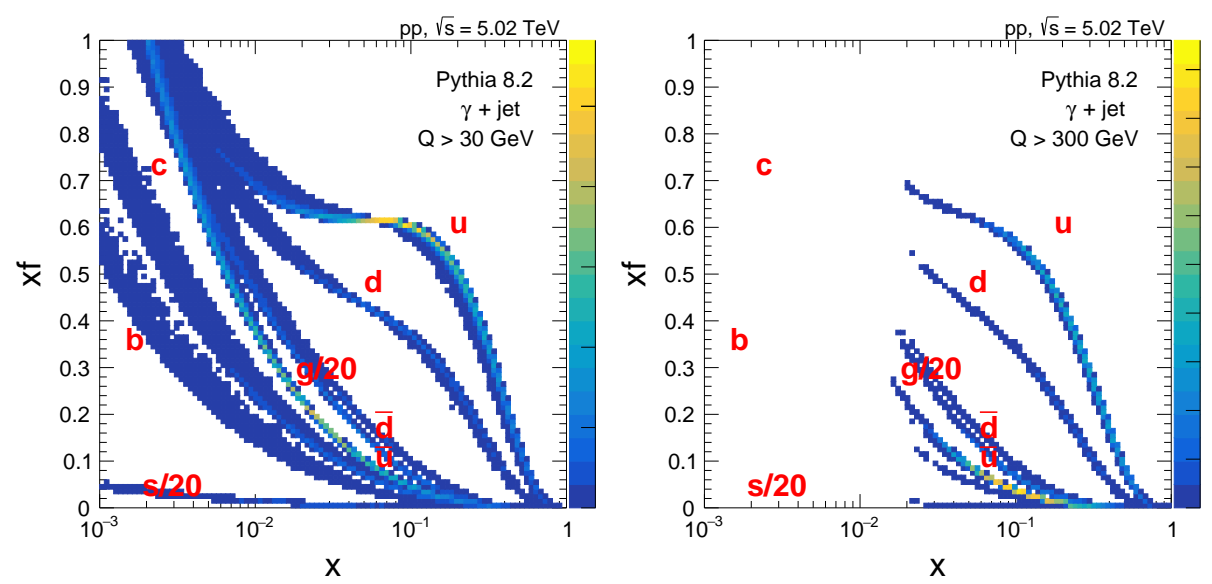

Figure 2: Parton density $x \cdot f\left(x, Q^{2}\right)$ versus momentum fraction $x$ of incoming partons weighted by their contribution to prompt photon processes in PYTHIA 8. Processes with momentum transfer $Q>30 \mathrm{GeV}$ and $Q>300 \mathrm{GeV}$ are on the left and right, respectively. Red labels mark the parton flavor contributing to a region. The $x \cdot f\left(x, Q^{2}\right)$ values are divided by 20 for gluons and $s$-quarks.

ticular $x>0.1$. Gluon densities vanish rapidly at even higher $x$, causing the dramatic decrease of quark-jet events.
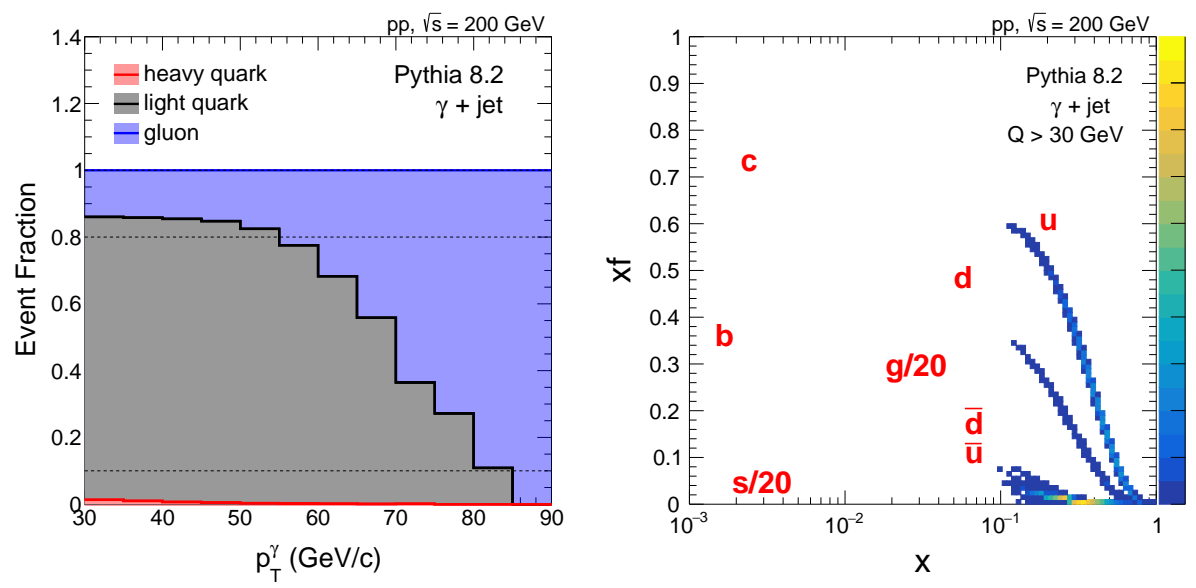

Figure 3: Left : Fig. 1 remade for pp collisions at $\sqrt{s}=200 \mathrm{GeV}$ and displaying a smaller $p_{\mathrm{T}}^{\gamma}$ range. Right : Fig. 2 remade for $\sqrt{s}=200 \mathrm{GeV}$.

\section{Momentum and angle correlations}

Effects of parton level processes in PYTHIA 8 on the $\gamma+$ jet observables are studied by comparing four scenarios. The first scenario is to switch off Multiparton interactions (MPI), the second is to switch off initial-state radiation (ISR), the third is to switch off final-state radiation (FSR). The fourth scenario is to switch on all of MPI, ISR, and FSR, which is the default setting in PYTHIA 8. 
Events are generated separately for each of the four scenarios. The left and right panel of Fig. 4 show the distributions of azimuthal angle difference, $\Delta \phi_{\mathrm{j} \gamma}$, and momentum imbalance, $x_{\mathrm{j} \gamma}$, respectively, for the four scenarios. The sharpest $\Delta \phi_{\mathrm{j} \gamma}$ and $x_{\mathrm{j} \gamma}$ distributions happen when ISR is switched off indicating that ISR is the dominant source of wide $\gamma+$ jet correlations.
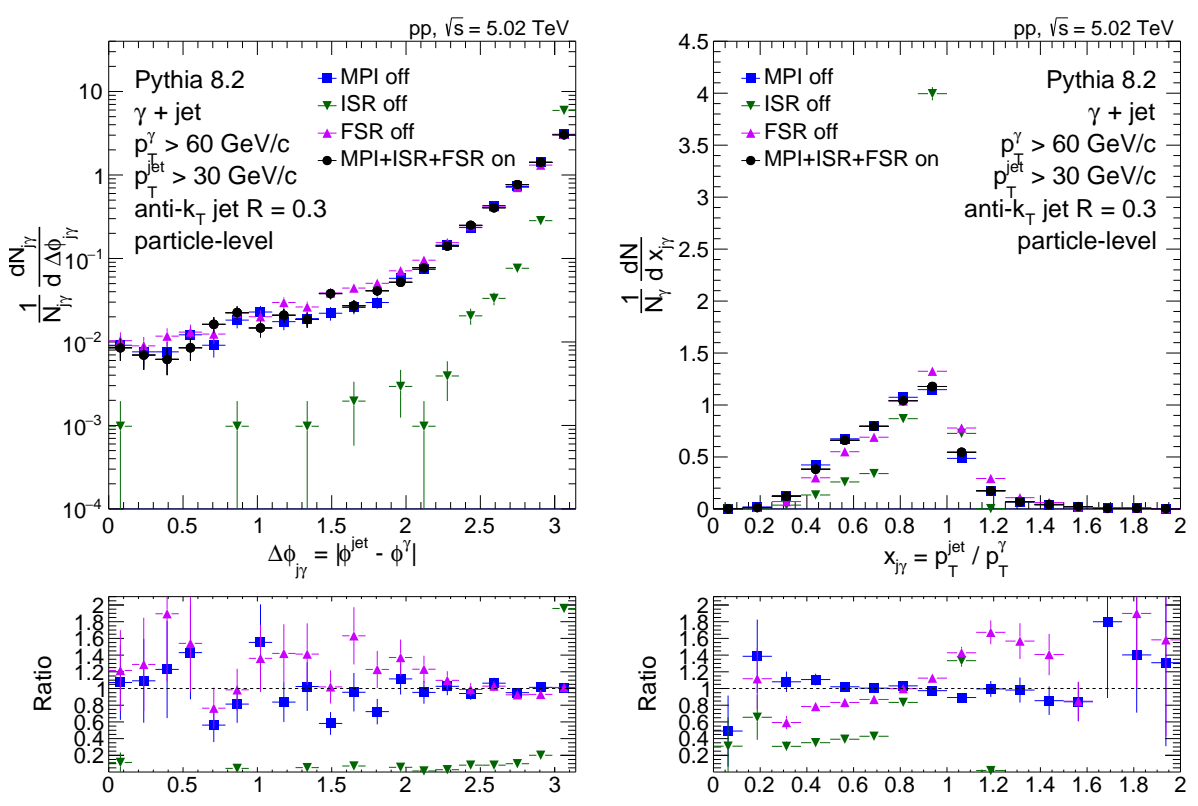

Figure 4: Top : Distributions of the azimuthal angle difference $\Delta \phi_{j \gamma}$ between the photon and the jet (left), and of the transverse momentum ratio $x_{\mathrm{j} \gamma}$ between the jet and the photon with $\Delta \phi_{j \gamma}>7 \pi / 8$ (right) for pp collisions at $\sqrt{s}=5.02 \mathrm{TeV}$. PYTHIA 8 events are generated separately by switching off the MPI (blue), ISR (green), FSR (violet), and by switching on all of MPI, ISR, and FSR (black). Bottom : Ratio of distributions from events with MPI (blue), ISR (green), FSR (violet) off to the distributions from events where all of MPI, ISR, and FSR are on.

The left panel of Fig. 5 shows the $\Delta \phi_{\mathrm{j} \gamma}$ distributions from the four scenarios at RHIC energies. ISR is the dominant source for the widening of $\Delta \phi_{j \gamma}$ at RHIC energies as well. Bottom-left panels of Fig. 4 and Fig. 5 show ratios of the $\Delta \phi_{j \gamma}$ from the first three scenarios to the one from the default setting, namely the fourth scenario. At LHC (RHIC) energies, the "ISR-off" and default settings differ by $\sim 100 \%(\sim 20 \%)$ at $\Delta \phi_{\mathrm{j} \gamma} \approx \pi$. Hence, ISR effects at RHIC energies are smaller than at LHC energies. This can be explained with the fact that a smaller phase space for ISR is available at RHIC because of the lower $\sqrt{s}$. The right panel of Fig. 5 compares the $\Delta \phi_{\mathrm{j} \gamma}$ for $\sqrt{s}=5.02 \mathrm{TeV}$ and $\sqrt{s}=200 \mathrm{GeV}$. The distribution for RHIC is much sharper because of smaller ISR effects.

\section{Angular resolution driven by the Underlying Event}

As briefly mentioned in Sec. 1, effects of the heavy-ion UE on a measurement can be reverted if the magnitude and direction of the effects are known. On the other hand, it is non-trivial to predict the direction if the measured quantity is vectorial. The UE effects on jet observables are studied via a toy UE. "Toy UE" particles are sampled randomly from HYDJET [13] events for the $10 \%$ most central $\mathrm{PbPb}$ collisions at $\sqrt{s_{\mathrm{NN}}}=5.02 \mathrm{TeV}$ and added to PYTHIA $8 \gamma+$ jet events. 

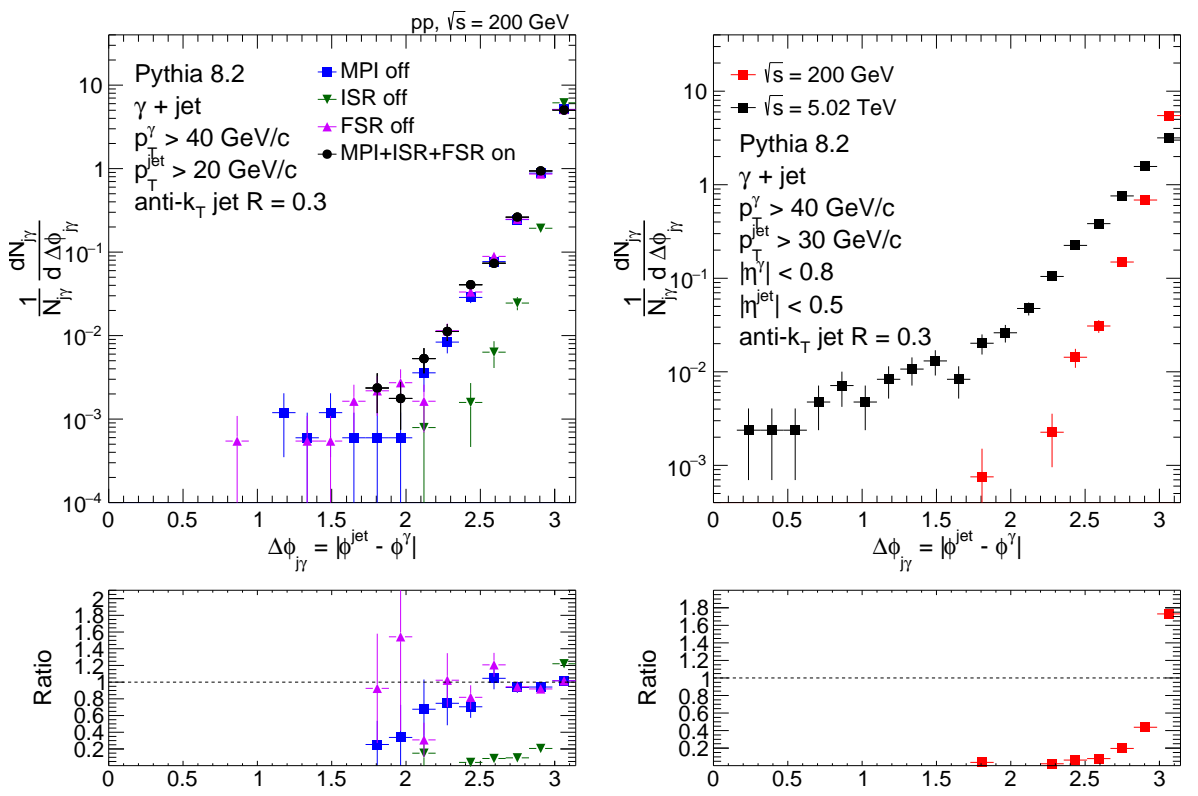

Figure 5: Top left : Distributions of the azimuthal angle difference $\Delta \phi_{\mathrm{j} \gamma}$ between the photon and and the jet for pp collisions at $\sqrt{s}=200 \mathrm{GeV}$. PYTHIA 8 events are generated separately by switching off the MPI (blue), ISR (green), FSR (violet), and by switching on all of MPI, ISR, and FSR (black). Top right : Comparison of $\Delta \phi_{j \gamma}$ for center-of-mass energies of $\sqrt{s}=5.02 \mathrm{TeV}$ (black) and $\sqrt{s}=200 \mathrm{GeV}$ (red) where same kinematic selections are used for both distributions. Bottom left : Ratio of distributions from events with MPI (blue), ISR (green), FSR (violet) off to the distributions from events where all of MPI, ISR, and FSR are on. Bottom right : Ratio of the distribution for $\sqrt{s}=200 \mathrm{GeV}$ to the one for $\sqrt{s}=5.02 \mathrm{TeV}$

Particles are clustered into jets using the anti- $k_{\mathrm{T}}$ algorithm in the FastJet framework $[14,15]$ with a distance parameter of $R=0.3$. The clustering is run separately for two cases, the first one uses only PYTHIA 8 particles (labelled as "Pythia"), the second one uses both toy UE particles and PYTHIA 8 particles (labelled as "Pythia+toy"). For the second case, the jet energy is corrected by subtracting the energy of the toy UE particles. Afterwards, the jet observables obtained from the two cases are compared. The left and right panels of Fig. 6 show this comparison for the jet fragmentation function observable $\xi^{\text {jet }}$, defined in Ref. [5], and the jet shape observable $\rho(r)$, defined in Ref. [7], respectively. $\xi^{\text {jet }}$ distributions are compatible for the two cases. In contrast, jet shapes for the "Pythia+toy" case are significantly wider than the "Pythia" case.

The sensitivity of the jet shapes to the inclusion of UE particles can be understood by studying how the observable is correlated with UE particles. Left (right) panel of Fig. 7 shows the jet shapes constructed using UE particles with $p_{\mathrm{T}}>1\left(p_{\mathrm{T}}>3\right) \mathrm{GeV} / \mathrm{c}$, for two different cases of particle selections. The first case (green) uses UE particles in the same event as the jet. The second case (red) uses UE particles from a randomly selected event (mixed event). The distributions increase monotonically with $r$. This is expected because the angular space increases with $r$ and for UE particles, any $(\eta, \phi)$ coordinate with respect to a jet is equally likely since jets and UE particles are intrinsically uncorrelated. The picture changes for particles with $p_{\mathrm{T}}>3 \mathrm{GeV} / \mathrm{c}$ where the distributions for the first case present a bias towards smaller $r$, and are even peaking in the 

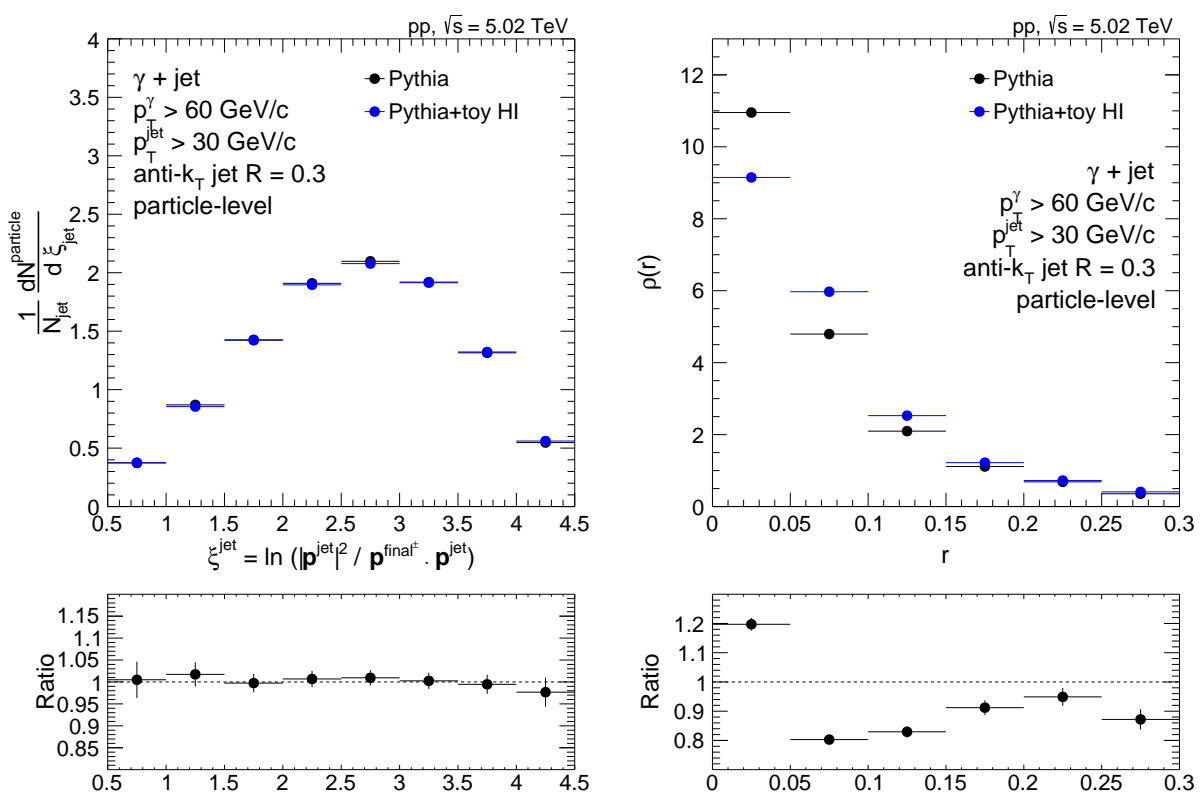

Figure 6: $\xi^{\text {jet }}$ distribution (left) and the differential jet shape $\rho(r)$ for jets associated with a photon in PYTHIA $8 \gamma+$ jet events where jets are clustered with the anti- $k_{\mathrm{T}}$ algorithm for $R=0.3$ using PYTHIA 8 particles (black), and using both PYTHIA 8 particles and toy UE particles (blue). Distributions are filled using charged PYTHIA 8 particles with $p_{\mathrm{T}}>1 \mathrm{GeV} / \mathrm{c}$.

$0.2<r<0.25$ bin. The shift towards smaller $r$ indicates that UE particles effect jet clustering in a way that the $(\eta, \phi)$ coordinate of the jet (jet axis) tends to be closer to that of the UE particles. The effect becomes larger with increasing energy of the UE particles and can be described as UE particles "pulling" the jet axis to themselves. The effected quantity is the jet $(\eta, \phi)$ coordinate, a vector quantity, whose direction of change is ambiguous, but correlated with the position of UE particles. A finely binned correction procedure is needed to revert the effects. An approach to overcome this effect without applying dedicated corrections would be to redefine the jet axis so that it becomes less sensitive to UE particles.

\section{Switch to Winner-take-all recombination scheme}

The standard jet axis is obtained from the $E$-scheme recombination scheme where two components are combined by adding up their 4-momenta [15]. Hence, the direction of the combined object is the momentum weighted average of the two components. Note that UE particles have relatively lower energy compared to particles from hard scattering. This implies that the sensitivity of the jet axis to UE can be reduced by using a recombination scheme that increases the weights of higher energy particles. The "winner-take-all" (WTA) recombination scheme [16, 17] takes the weight increase to an extreme such that the combined object is assigned the $(\eta, \phi)$ coordinate of the component with highest $p_{\mathrm{T}}$.

The jet fragmentation function and jet shape observables in Fig. 6 were constructed using jets whose constituents are recombined using the $E$-scheme. The same observables are extracted using 

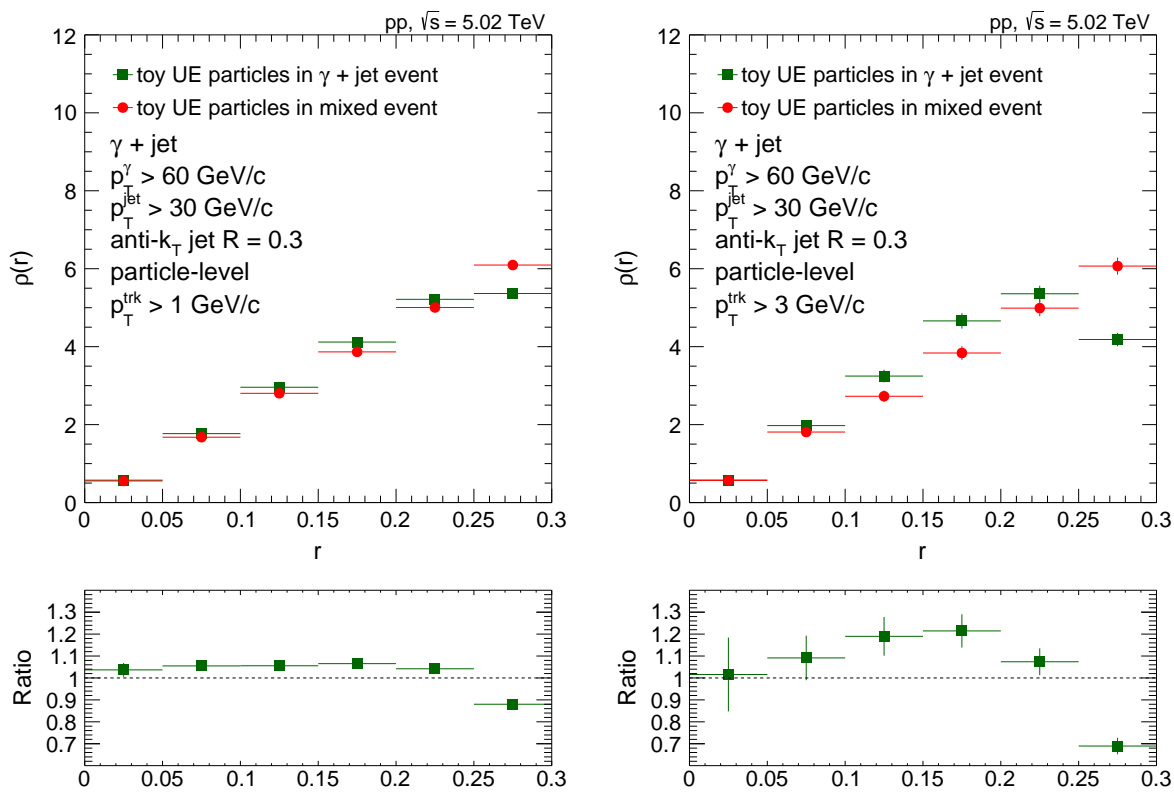

Figure 7: The differential jet shape $\rho(r)$ constructed using charged UE particles in the same event as the jet (green) and using charged UE particles from a randomly selected event (red). Particles on the left and right panels satisfy $p_{\mathrm{T}}>1 \mathrm{GeV} / \mathrm{c}$ and $p_{\mathrm{T}}>3 \mathrm{GeV} / \mathrm{c}$, respectively.

jets recombined with the WTA scheme and shown in Fig. 8. The $\xi^{\text {jet }}$ distributions for the "Pythia" and "Pythia+toy" cases are similar as in the E-scheme case. This time, jet shapes are also in good agreement for the two cases and this is where the WTA scheme becomes favorable. The jet axis determined using the WTA scheme is more robust against the heavy-ion UE as it is reducing the UE sensitivity of substructure observables that are based on jet angle.

The WTA scheme can promise benefits not only for jet substructure studies, but also for angular correlations. It is hypothesized that the medium-induced $p_{\mathrm{T}}$ broadening of partons [18] would widen the azimuthal correlations between the two hard scatterers [19, 20]. Jet quenching has been observed at the LHC, but no significant medium effect was seen in azimuthal angle correlations $[21,22,23,1,2,24,25]$. It is possible that the medium-induced widening of angular correlations are undermined by the large widening caused by vacuum radiation processes [20]. The widening from vaccum processes is smaller at lower collisions energies, as was shown in the right panel of Fig. 5. Therefore, the medium-induced $p_{\mathrm{T}}$ broadening is expected to reveal itself at RHIC energies in a relatively stronger way than at LHC energies. However, the effect on azimuthal correlations can still be small, implying that a good experimental control of the jet angle will be vital for $p_{\mathrm{T}}$ broadening studies.

The exercise with toy UE particles, explained in Sec. 4, has been repeated for RHIC energies. Jet clustering is run for the "Pythia" and "Pythia+toy" cases. Fig. 9 shows the distributions of azimuthal angle difference, $\Delta \phi_{j \gamma}$, for the two cases. For each case, jet clustering is run separately with the $E$-scheme (left panel) and the WTA recombination schemes (right panel). For jets recombined with the $E$-scheme, the toy UE causes a roughly $5 \%$ deviation at $\Delta \phi_{\mathrm{j} \gamma} \approx \pi$, whereas for jets with the WTA scheme the deviation at the same $\Delta \phi_{\mathrm{j} \gamma}$ is smaller than $1 \%$. The ratios between the $\Delta \phi_{\mathrm{j} \gamma}$ 

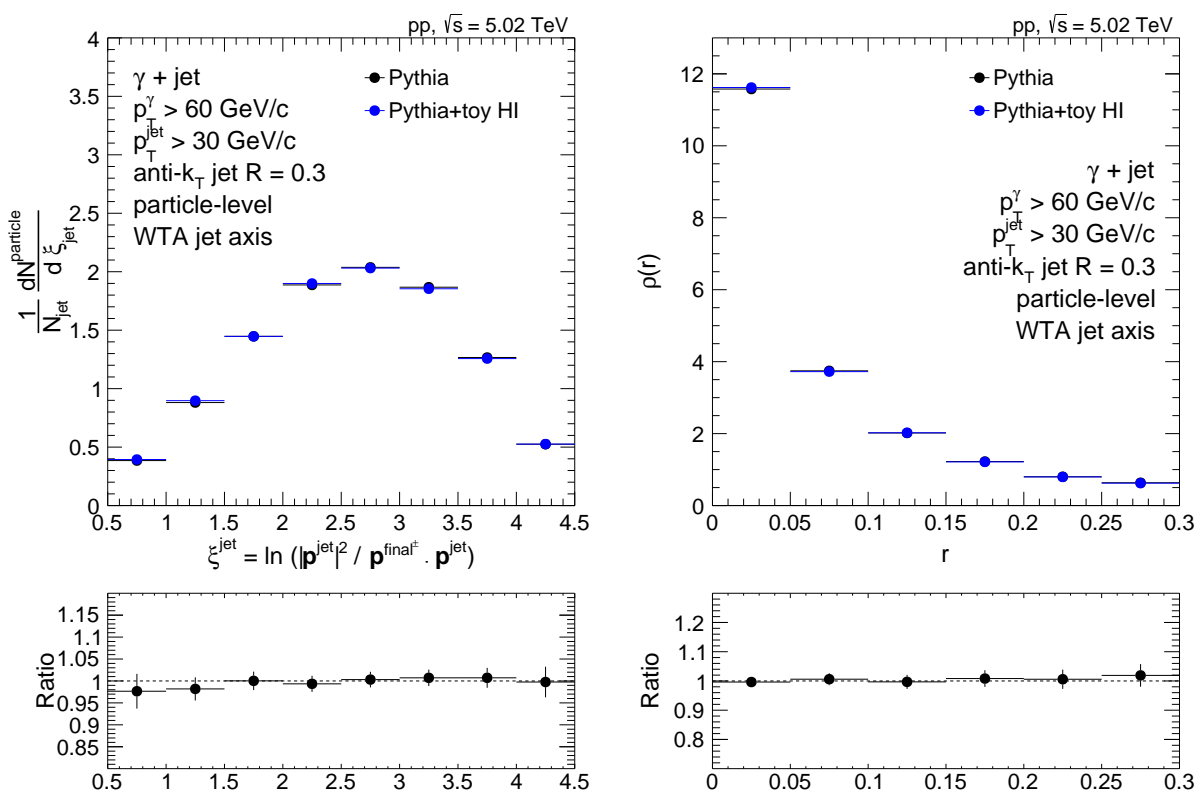

Figure 8: Fig. 6 remade using jets whose constituents are recombined with WTA scheme.

distributions from "Pythia" and "Pythia+toy" cases are shown for the $3 \pi / 4<\Delta \phi_{j \gamma}<\pi$ range. Distributions are much more consistent when using the WTA scheme. Apparently, the WTA scheme reduces distortions of the jet angle caused by the UE.

\section{Summary}

The lack of strong interaction between electroweak bosons and the QCD matter make $\gamma+$ jet events precise probes to study parton modifications in medium. Measurements from the LHC experiments showed that the momentum and angle correlations between $\gamma+$ jet pairs are wide even in pp collisions where no large medium is expected to form. Simulation of PYTHIA 8 events showed that the widening of correlations is caused by parton level processes in vacuum, ISR being the dominant source of widening. The phase space for ISR shrinks with decreasing collision energy making the widening effects smaller.

Reverting the effects of heavy-ion UE on a vector quantity is challenging due to a lack of knowledge about the direction of the effects. When jets are clustered using the standard recombination scheme ( $E$-scheme), the UE particles can distort the jet $(\eta, \phi)$ coordinate in a way correlated with their kinematics, making the jet angle determination sensitive to the UE. The sensitivity can be significantly reduced if jets are clustered using the WTA recombination scheme. Simulation of PYTHIA 8 and toy UE events suggest that the WTA scheme can be particularly advantageous for angle dependent jet substructure measurements and for studies of medium-induced $p_{\mathrm{T}}$ broadening via azimuthal correlations. 

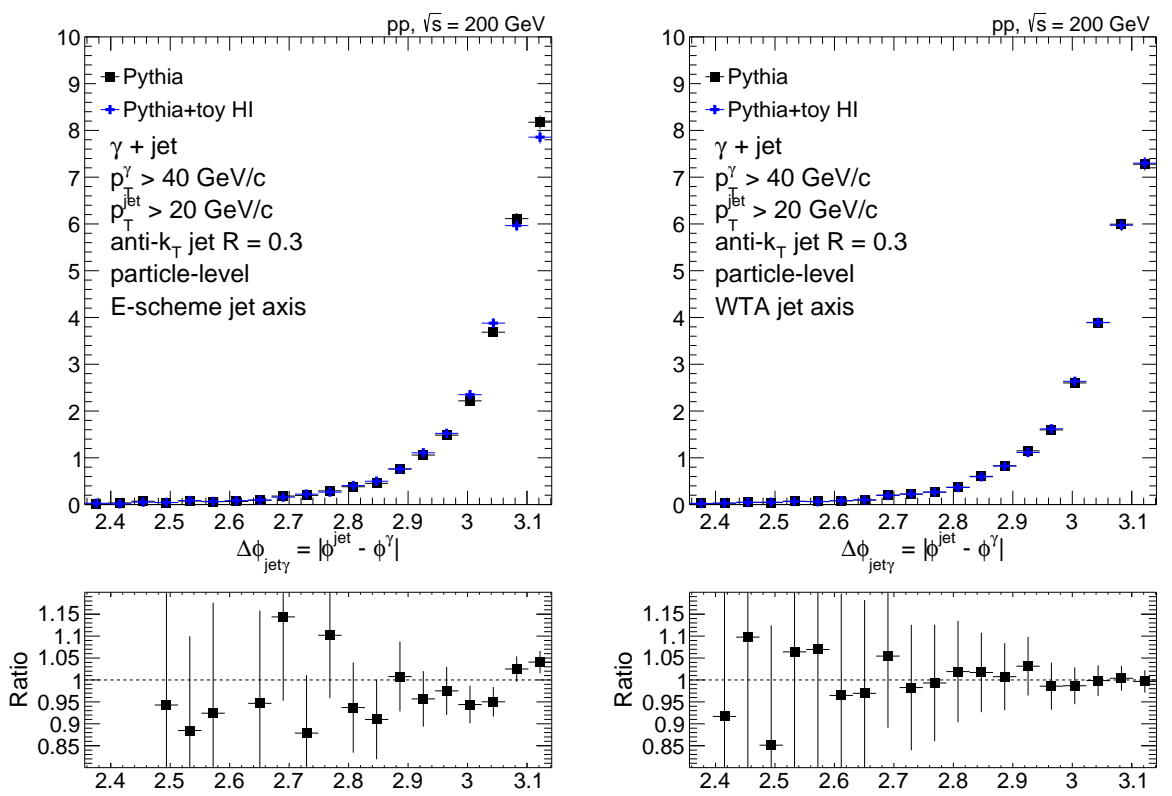

Figure 9: Distributions of the azimuthal angle difference $\Delta \phi_{\mathrm{j} \gamma}$ between the photon and the jet in PYTHIA $8 \gamma+$ jet events for pp collisions at $\sqrt{s}=200 \mathrm{GeV}$. Jets are clustered using PYTHIA 8 particles (black), and using both PYTHIA 8 particles and toy UE particles (blue) (See Sec. 4). Jets are recombined using the $E$-scheme (left) and the WTA scheme (right).

\section{References}

[1] CMS collaboration, Studies of jet quenching using isolated-photon+jet correlations in PbPb and pp collisions at $\sqrt{s_{\mathrm{NN}}}=2.76 \mathrm{TeV}$, Phys. Lett. B 718 (2013) 773 [1205.0206].

[2] CMS collaboration, Study of jet quenching with isolated-photon+jet correlations in PbPb and pp collisions at $\sqrt{s_{\mathrm{NN}}}=5.02 \mathrm{TeV}$, Phys. Lett. B 785 (2018) 14 [1711.09738].

[3] ATLAS collaboration, Measurement of photon-jet transverse momentum correlations in $5.02 \mathrm{TeV} \mathrm{Pb}$ + Pb and pp collisions with ATLAS, Phys. Lett. B 789 (2019) 167 [1809.07280].

[4] CMS collaboration, Study of jet quenching with $\mathrm{Z}+$ jet correlations in $\mathrm{Pb}-\mathrm{Pb}$ and pp collisions at $\sqrt{s_{\mathrm{NN}}}=5.02 \mathrm{TeV}$, Phys. Rev. Lett. 119 (2017) 082301 [1702.01060].

[5] CMS collaboration, Observation of Medium-Induced Modifications of Jet Fragmentation in $\mathrm{Pb}-\mathrm{Pb}$ Collisions at $\sqrt{s_{\mathrm{NN}}}=5.02 \mathrm{TeV}$ Using Isolated Photon-Tagged Jets, Phys. Rev. Lett. 121 (2018) 242301 [1801.04895].

[6] ATLAS collaboration, Comparison of fragmentation functions for light-quark- and gluon-dominated jets from pp and Pb+Pb collisions in ATLAS, Submitted to: Phys. Rev. Lett. (2019) [1902.10007].

[7] CMS collaboration, Jet Shapes of Isolated Photon-Tagged Jets in Pb-Pb and pp Collisions at $\sqrt{s_{\mathrm{NN}}}=5.02 \mathrm{TeV}$, Phys. Rev. Lett. 122 (2019) 152001 [1809.08602].

[8] CMS collaboration, Modification of jet shapes in PbPb collisions at $\sqrt{s_{\mathrm{NN}}}=2.76 \mathrm{TeV}$, Phys. Lett. B 730 (2014) 243 [1310.0878].

[9] CMS collaboration, Measurement of transverse momentum relative to dijet systems in $\mathrm{PbPb}$ and $\mathrm{pp}$ collisions at $\sqrt{s_{\mathrm{NN}}}=2.76 \mathrm{TeV}$, JHEP 01 (2016) 006 [1509.09029]. 
[10] CMS collaboration, Correlations between jets and charged particles in $\mathrm{PbPb}$ and pp collisions at $\sqrt{s_{\mathrm{NN}}}=2.76 \mathrm{TeV}$, JHEP 02 (2016) 156 [1601.00079].

[11] CMS collaboration, Jet properties in PbPb and pp collisions at $\sqrt{s_{\mathrm{NN}}}=5.02 \mathrm{TeV}$, JHEP 05 (2018) $006[1803.00042]$.

[12] T. Sjöstrand, S. Ask, J. R. Christiansen, R. Corke, N. Desai, P. Ilten et al., An Introduction to PYTHIA 8.2, Comput. Phys. Commun. 191 (2015) 159 [1410.3012].

[13] I. P. Lokhtin and A. M. Snigirev, A model of jet quenching in ultrarelativistic heavy ion collisions and high- $p_{\mathrm{T}}$ hadron spectra at RHIC, Eur. Phys. J. C 45 (2006) 211 [hep-ph/ 0506189 ].

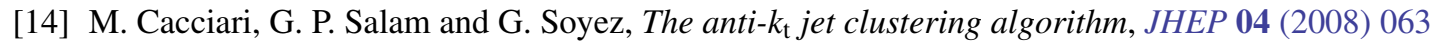
[0802.1189].

[15] M. Cacciari, G. P. Salam and G. Soyez, FastJet user manual, Eur. Phys. J. C 72 (2012) 1896 [1111.6097].

[16] D. Bertolini, T. Chan and J. Thaler, Jet Observables Without Jet Algorithms, JHEP 04 (2014) 013 [1310.7584].

[17] A. J. Larkoski, D. Neill and J. Thaler, Jet Shapes with the Broadening Axis, JHEP 04 (2014) 017 [1401.2158].

[18] R. Baier, Y. L. Dokshitzer, A. H. Mueller, S. Peigne and D. Schiff, Radiative energy loss and $p_{\perp}$-broadening of high energy partons in nuclei, Nucl. Phys. B 484 (1997) 265 [hep-ph/9608322].

[19] A. H. Mueller, B. Wu, B.-W. Xiao and F. Yuan, Probing Transverse Momentum Broadening in Heavy Ion Collisions, Phys. Lett. B 763 (2016) 208 [1604.04250].

[20] L. Chen, G.-Y. Qin, S.-Y. Wei, B.-W. Xiao and H.-Z. Zhang, Probing Transverse Momentum Broadening via Dihadron and Hadron-jet Angular Correlations in Relativistic Heavy-ion Collisions, Phys. Lett. B 773 (2017) 672 [1607.01932].

[21] CMS collaboration, Observation and studies of jet quenching in PbPb collisions at $\sqrt{s_{\mathrm{NN}}}=2.76 \mathrm{TeV}$, Phys. Rev. C 84 (2011) 024906 [1102.1957].

[22] ATLAS collaboration, Observation of a centrality-dependent dijet asymmetry in lead-lead collisions at $\sqrt{s_{\mathrm{NN}}}=2.76 \mathrm{TeV}$ with the ATLAS detector at the LHC, Phys. Rev. Lett. 105 (2010) 252303 [1011.6182].

[23] CMS collaboration, Jet momentum dependence of jet quenching in PbPb collisions at $\sqrt{s_{\mathrm{NN}}}=2.76 \mathrm{TeV}$, Phys. Lett. B 712 (2012) 176 [1202.5022].

[24] ALICE collaboration, Measurement of jet quenching with semi-inclusive hadron-jet distributions in central Pb-Pb collisions at $\sqrt{s_{\mathrm{NN}}}=2.76 \mathrm{TeV}$, JHEP 09 (2015) 170 [1506.03984].

[25] CMS collaboration, Comparing transverse momentum balance of b jet pairs in $p p$ and $\mathrm{PbPb}$ collisions at $\sqrt{s_{\mathrm{NN}}}=5.05 \mathrm{TeV}$, JHEP 03 (2018) 181 [1802.00707]. 